\title{
Bats as A Viral Reservoir: A Short Review Of Their Ecological Characters and Immune System
}

\author{
Desrayni Hanadhita $^{\text {b) }}$, Aryani Sismin Satyaningtijas ${ }^{\text {c) }}$, Srihadi Agung Priyono ${ }^{\text {a) }}$ \\ Department of Anatomy Physiology and Pharmacology, Faculty of Veterinary Medicine, \\ Bogor Agricultural University (IPB), Bogor 16680, Indonesia \\ ${ }^{a)}$ Corresponding author: ysrihadi@ipb.ac.id \\ b)desraynihanadhita@gmail.com \\ ${ }^{c)}$ niekesis@yahoo.co.id
}

\begin{abstract}
Bats are reported as natural reservoir host of several viruses, many of which cause severe human disease. Infected bats usually do not show any clinical symptoms and persistently infected. Bats immunity against viruses is an evolutional process of self-defense created by ecological adaptation between host and pathogen. Bats have unique characters which support them as a viral reservoir, whereas RNA viruses which are commonly carried by bats take an advantage of these characteristics to survive. The role of bats as reservoirs is also supported by several differences of immune system than other mammals. Several studies on molecular factors of bats immune system lead to a hypothesis that bats are able to control viral replication very early in the immune response through innate antiviral mechanism. However, to complete understanding of the nature of immune responses of bats to the virus still needs a lot of study. Advance technology on molecular techniques revealed the immune factors and provided numerous data about molecular factors in bat immunity, however comprehensive studies about morphology of bats immune organs are still lacking. One of the important immune organs which have functional variations due to their structural differences between species is the spleen, which will be our main focus of study in the future. We hope that the result of our study will be able to provide the database on the morphology and physiology of bat spleen and give better understanding on bats immune system.
\end{abstract}

Keywords: chiropterans, reservoir, flying mammal, zoonosis, immunology

\section{INTRODUCTION}

Bats are the primary source of zoonotic viruses throughout the world. Molecular studies show that bats are a natural reservoir of rabies and other Lyssavirus (Kuzmin et al. 2011). Bats were also reported as a major reservoir for some fatal zoonotic virus like sudden acute respiratory syndrome (SARS) like coronavirus (Wang et al. 2006) and Middle East respiratory syndrome coronavirus (MERS-CoV) (Mohd et al. 2016); Ebola and Marburg hemorrhagic fever filovirus (Briand et al. 2014); and paramyxovirus cause Nipah (Looi\& Chua 2007) and Hendra (Smith \& Wang 2013). Bats infected with virus do not show any clinical symptoms and in some cases they become persistently infected (Baker and Zhou 2015). A comparative study was conducted by Luis et al. (2013) who described that bats are more infected by zoonotic virus per species than rodents, although the number of rodent 
species twice as much of the bat. This study demonstrates that bats should be considered as a unique source of emerging zoonotic diseases.

Low virulence accompanied by a high diversity of viruses lead to a perception that bats have a unique and more effective mechanism to regulate viral replication than other mammals (Calisher et al. 2006; Wang et al. 2011; Baker \& Zhou 2015; O'Shea et al. 2014; Schountz 2014; Moratelli and Calisher 2015). The ecological balance between pathogens and their hosts that maintain infection and transmission in the absence of clinical symptoms can contribute to the evolution of both (Wang et al. 2011). Whole-genome sequence analysis of bats has showed a genetic change which may contribute to bats evolution and their ability to combat virus (Zhang et al. 2013). Bat's immune system must detect and eliminate pathogens to prevent the disease meanwhile the viruses must maintain the replication and transmission above the extinction threshold (Real \&Biek 2007). Virus requires several criteria in order to survive such as: (1) they have a capability to infect and survive inside the host without causing any substantial diseases and (2) they must be transmitted to other vulnerable host before eliminated by immune systems or the host died from infection (Schountz 2014). The phenomenon of the viral persistency without causing any damage in the bat's body may lead to the evolution of self-defense that was created by the ecological adaptation between host and pathogens (Wang et al. 2011).

\section{ECOLOGICAL CHARACTERS OF BATS AS A VIRAL RESERVOIR}

Bats belong to order Chiropteran which is divided into two suborders: Yinpterochiroptera (known as megachiroptera or megabats) and Yangochiroptera (known as microchiropteraataumicrobats) (Telling et al. 2005). Chiropterans are the most successful mammalian suborder in order to survive and they have several characters which explained the viral diversity that they carried. Chiropteran order consisted of 1,200 species representing $20 \%$ of mammal species (Altringham 2011). The vast numbers of species provide great breeding sites of the virus. Bats are the only mammals that have an ability to fly and several species of bats can fly very far from their hibernation sites. The ability to fly provides a tremendous opportunity to have a direct and indirect interaction with other animal species in different geographic areas which increased interspecies viral transmission (Wang et al. 2011). Bats flying ability also influence the immune response by raising the metabolic rate up to 1516 times that elevated body temperature and can be categorized as "fever" (O'Shea et al. 2014). Fever influenced immune response by increased innate and adaptive immune system and suppresses viral proliferation (Hasdayet al. 2000; Launey et al. 2011).

Some bats species can undergo hibernation and torpor state which decrease body temperature and metabolic rate. Low body temperature and metabolic rate lead to decrease of immune response and prevent viral destruction from bat populations (Calisher et al. 2006; Wang et al. 2011; Moratelli\& Calisher 2015). Bats have a roosting habit with a high density and converge with diverse species which can increase the possibility of viral transmission intra- and interspecies (Luis et al. 2013). Bats have a very long life span compared to the land mammals the same body size (South \& Wilkinson 2009). Many species of the Yangochiroptera or Microchiroptera have a life span up to 35 years which documented in 
brown bat (Calisher et al. 2006). Long life span allows persistent infection of virus among bats and increases a possibility to spread to other vertebrates.

Viral transmissions between bats and domestic animals or human are supported by bats special characteristics. Fruit bats cannot swallow food in a large size therefore they chew and spit it out. The remnant fruit can be ingested by other animal species and may become infected by viral particles in bat saliva (Dobson 2005). Insectivorous bats use echolocation abilities to navigate their path. Ultrasonic waves generated from the vibrations of the larynx can spray aerosol of larynx secrete and transmitted virus to other species (Calisher et al. 2006). Feces and urine droplet from bats while flying or roosting was also becoming a transmission media. Viral spread from bats infected vulnerable host and they transmit it to the human lead to zoonosis diseases (Smith \& Wang 2013; Mohd et al. 2016).

\section{VIRAL ADAPTION IN BATS INFECTION}

Wang et al. (2011) described the characteristics of virus infection in bats is not an implementation of effective host immune responses, but rather the absence of pathological processes as a result of a highly evolved viral survival strategy. Zoonotic viruses found in bats are particularly RNA virus that replicates in the cytoplasm which has a high potential rate of replication, mutation frequency, and recombination (Luis et al. 2013). Viruses with high adaptability which persistently infects bat populations were also assumed to keep the bats population from predators and acts as a biological weapon for bats (Wang et al. 2011). The best defense weapon is not intrusive to host and released when the host is threatened. Henipavirus is one of the viruses in bat population with the low titer and not harmful to bats. Virus can be transmitted to other animal species that have fatal consequences for the majority of vertebrates when host being threatened (Chua 2003). Bats suffered such an extreme ecological changes when migrating and hibernate which lead to a strong formation of viral defense in a wide temperature range and thus potentially cause disease in a new host species (O'Shea et al. 2014). The completions of viral characteristics during the evolutionary history of bats were assumed to support the success of virus survival strategy and made the bats as their natural reservoir (Wang et al. 2011).

\section{THE IMMUNE SYSTEM OF BATS}

Bats have several immune elements similar to other mammals' species which includes pattern recognition receptors, interferon, complement activity, immunoglobulin, antibody response, major histocompatibility complex (MHC), cytokine, and $\mathrm{T}$ cells dependent immunity but they also have several differences which support their ability to resist viral infection (Baker \& Zhou 2015). Baker and Zhou (2015) summarized the immune system of bats and hypothesized that bats can resist viral infection in an early stage through their innate immune system. The differences of innate immune system between bats and other mammals that their toll-like receptor (TLR) have additional TLR 13 which previously only identified in rodent and bovine (Cowled et al. 2011; Zhang et al. 2013) and bats have more IFN $\omega$ and IFN $\delta$ gene but less IFN $\alpha$ gene from other mammals (Kepler et al. 2010). They also have the unique adaptive immunity elements such as: wide varieties of heavy chain variable (VH) 
gene at their antibody (Baker et al. 2010); the unique structure of peptide binding groove in class I MHC (Baker and Zhou 2015); and the different DR beta locus polymorphism in class II MHC in every different species of bats (Richman et al. 2010; Mayer and Brunner 2007; Schad et al 2011).

Unique bats immune systems elements were assumed supports bats resistance to virus. However there is a lot of study needed to understand the nature of bats immune systems in viral infection defense. Advance technology on molecular techniques revealed the immune factors and provided numerous data about molecular factors in bat immunity, however comprehensive studies about morphology of bats immune organs are still lacking. One of the important immune organs which have functional variations due to their structural differences between species is the spleen, which will be our main focus of study in the future. Spleen is a largest lymphoid organ in the body. It does not have any afferent lymphatic vessels therefore it directly filter the blood from systemic circulation.Spleen vascular structures are supporting it function as filtration, immunologic, storage, and hematopoiesis. Spleen is the unique lymphoid organ that has different structures and resident cells include: shape and orientation; fibrous tissue and elastic fibers distributions; blood circulation type; sinusoid type; the characteristics of white pulp (periarteriolar lymphatic sheaths (PALS) morphology, follicle, and marginal zone with the resident cells); the characteristics of periarteriolar macrophage sheaths (PAMS) or ellipsoid; and the characteristics blood spleen barrier. The differences of structures and resident cells of the spleen will influence its physiological function between species (Haley 2003; Cesta 2006). Our preliminary study showed the spleen weight ratio to body weight of fruit bats were $0.4 \%$ which is slight larger than rat $(0.2 \%)$ and apparently smaller than mice $(0.7 \%)$ (Webster danLiljegren 1955; Cesta 2006).However, bats as viral reservoir have a unique lymphocyte population in the spleen which is different from other mammals, and dominated by CD8+ T cells rather than CD4+ T cells. The large populations of CD8+ T cells may be indicate that bat's spleen always in the steady state to combat virus (Gomez et al. 2016). However morphological study of the spleen of bats must be conducted, for complete understanding of the physiological function.

\section{CONCLUSION}

Resilience of the bats against viral infection has a great association with their ability to maintain ecological balance between their immune system and high adaptability of viruses that infect them. Ecological adaptations that occur throughout the evolutionary history of bats affect their immune elements that support their resistance to viruses.Our preliminary study showed the spleen weight ratio to body weight of fruit bats were $0.4 \%$. The differences of structures and resident cells of the spleen will influence its physiological function. Our future study about morphophysiology of bat's spleen is expected to describe the role of bat's spleen in viral infections. We hope our results of study can provide a database of the morphology and physiology of bat's spleen and give a better understanding to the immune system of bats. 


\section{REFERENCES}

1. A.D.Luis et al., Proc. R. Soc. Lond [Biol], 280, 1-9 (2013).

2. A.P.Dobson, Sciencemagazine, 310, 628-629 (2005).

3. C.H.Calisher et al., Clin. Microbiol. Rev., 19(3), 531-545 (2006).

4. C.M.Cowledet al., Dev.Comp. Immunol., 35, 7-18 (2011).

5. E.C.Telling, Science, 307, 580-584 (2005).

6. F. Mayer \& A.Brunner, Heredity99, 257-264. (2007).

7. G. Zhang et al., Science, 339, 456-459 (2013).

8. H.A.Mohdet al., Virol. J.13(87), (2016).

9. I.Smith \&L.F.Wang, Curr. Opin. Virol.,3, 84-91 (2013).

10. I.V.Kuzminet al., Emerg. Health Threats J.,4, 1-17 (2011).

11. J.Schad, et al., Heredity, 107, 115-126 (2011).

12. J.D. Altringham. Bats From Evolution to Conservation. (Oxford University Press Inc., New York, 2011).

13. J.M Gomez. et al., Nature, 6(37796), (2016).

14. J.M.South \&G.S.Wilkinson Ageing Res. Rev., 237, 1-8 (2009)..

15. K,B. Chua, J. Clin. Virol.,26(3), 265-275 (2003).

16. L.A.Real \&R.Biek, Curr. Top. Microbiol. Immunol.,315, 33-49 (2007).

17. L.F.Wang et al., Emerg. Infect. Diseases, 12(12), 1834-1840 (2006).

18. L.M.Looi\&K.B.Chua, Malays. J. Pathol., 29(2), 63-67 (2007).

19. M.F.Cesta, Toxicol. Pathol.,34, 455-465 (2006).

20. M.L. Baker \& P.Zhou, "Bat Immunology", in Bats and Viruses: A New Frontier of Emerging Infectious Diseases, edited by L.F. Wang and C. Cowled(John Wiley \& Sons, Inc., New Jersey, 2015), pp. 327-348.

21. M.L.Baker et al., Immunogenetics, 62, 173-184 (2010).

22. P. Zhouet al., J. Immunol., 186, 3138-3147 (2011).

23. R.Moratelli\&C.H. Calisher, Mem. Inst.Oswaldo Cruz, 110(1), 1-22 (2015).

24. Richman et al., Int J Immunogenet, 37, 401-405 (2010).

25. S.Briandet al., N. Engl. J. Med., 371, 1180-1183 (2014).

26. S.H. Webster \&E.J. Liljegren, Dev. Dyn, 97(1), 129-153 (1955).

27. T.Schountz, Viruses, 6, 4880-4901 (2014).

28. TKepleret al., BMC Genomics, 11, 444 (2010).

29. T.J.O'Shea et al., Emerg. Infect. Diseases, 20 (5), 741-745 (2014).

30. Y.Launeyet al., Crit. Care, 15, 222-229 (2011). 